\title{
Um Modelo para Recomendação de Objetos de Aprendizagem Utilizando Similaridade de Sessões
}

\author{
A Model for Learning Object Recommendation Using Similarity of Sessions
}

\author{
Tiago Wiedemann \\ Universidade do Vale do Rio dos \\ Sinos - UNISINOS \\ Programa de Pós Graduação em \\ Computação Aplicada \\ Av. Unisinos, 950 \\ São Leopoldo - RS - Brasil \\ tiagowrs@gmail.com
}

\author{
Jorge L. V. Barbosa \\ Universidade do Vale do Rio dos \\ Sinos - UNISINOS \\ Programa de Pós Graduação em Com- \\ putação Aplicada \\ Av. Unisinos, 950 \\ São Leopoldo - RS - Brasil \\ jbarbosa@unisinos.br
}

\author{
Sandro J. Rigo \\ Universidade do Vale do Rio dos \\ Sinos - UNISINOS \\ Programa de Pós Graduação em \\ Computação Aplicada \\ Av. Unisinos, 950 \\ São Leopoldo - RS - Brasil \\ rigo@unisinos.br
}

\begin{abstract}
Resumo Um Objeto de Aprendizagem (OA) é qualquer entidade ou recurso que possa ser utilizado no aprendizado auxiliado por computador. Podem ser textos, conteúdo multimídia, apresentações, programas ou qualquer outro tipo de conteúdo digital, normalmente disponibilizados em portais Web ou em sistemas de Ensino A Distância (EAD). Os OAs consultados por um aluno durante uma sessão de acesso a estes portais, possuem relação com os interesses de pesquisa do aluno durante a duração da sessão. Este artigo propõe um modelo para recomendação que explora esta relação, ao recomendar OAs baseando-se na análise de similaridade entre históricos de sessões. O modelo proposto recebe a sequência de OAs consultados durante a sessão atual do usuário e localiza sessões cujas sequências de OAs consultados sejam similares à sequência da sessão corrente. Os OAs encontrados nas sessões similares são então recomendados ao usuário. Foi desenvolvido um protótipo aplicado a um cenário real e os resultados alcançados mostraram a viabilidade da proposta.
\end{abstract}

Palavras-Chave: Sistemas de Recomendação, Análise de Similaridade, Filtragem Colaborativa, Objetos de Aprendizagem

\begin{abstract}
A learning object (LO) is any entity or resource that can be used in computer-aided learning. Can be text, multimedia content, presentations, programs or any other type of digital content, usually available on web portals or distance learning systems. The LOs consulted by a student during a session of access to these portals are related to the research interests of the student for the duration of the session. This article proposes a model for recommending that explores this relationship by recommending LOs based on the analysis of similarity between historical sessions. The proposed model receives the sequence of LOs consulted during the current user session and sessions located whose sequences are similar to LOs consulted following the current session. LOs found in similar sessions are then recommended to the user. A prototype applied to a real scenario was developed and the results obtained showed the feasibility of the proposal .
\end{abstract}

Keywords: Recommender Systems, Similarity Analysis, Collaborative Filtering, Learning Objects 


\section{Introdução}

Um Objeto de Aprendizagem (OA) é qualquer entidade ou recurso que possa ser utilizado no aprendizado auxiliado por computador. Podem ser textos, conteúdo multimídia, apresentações, programas ou qualquer outro tipo de conteúdo digital. O objetivo da disponibilização destes objetos na web é a reutilização dos mesmos em diferentes contextos educacionais, reduzindo os custos de sua produção [1].

Com o aumento da quantidade de OAs disponibilizados, assim como o próprio crescimento da web, tornou-se necessário a criação de bancos de dados organizados para seu armazenamento e catalogação. Estes bancos de dados disponíveis na web são chamados de "Repositórios" [2]. Os repositórios permitem a disponibilização de OAs para estudantes e educadores de forma individual ou organizados em grupos temáticos. Um exemplo de repositório é o Banco Internacional de Objetos Educacionais [3], mantido pelo MEC.

Porém, mesmo com a organização dos OAs em repositórios dedicados, a localização de objetos que atendam as necessidades dos usuários ainda é uma tarefa complexa, em virtude do volume de informações. Para solucionar este problema surgiram os sistemas de recomendação, que visam auxiliar a busca por OAs que se adequem ao perfil do usuário [4]. Por perfil de usuário, entende-se uma estrutura que contenha informações sobre as preferências, comportamento e contexto do usuário [5]. De maneira geral um sistema de recomendação é capaz de indicar recursos úteis aos usuários ou grupos, através de uma avaliação que procura determinar o quão útil uma determinada indicação é para o usuário [6].

$\mathrm{Na}$ área educacional, a utilidade de uma indicação ou recurso pode ser estimada a partir do conteúdo de cada objeto em conjunto com o perfil do usuário que está realizando a consulta. Por exemplo, Al-Khalifa [7] propõe um algoritmo de recomendação que analisa o perfil de cada usuário em conjunto com o histórico de todos os OAs já acessados pelo mesmo e o ranking dado pelo usuário a cada objeto.

No entanto, a análise baseada no perfil considera todo o histórico de OAs consultados pelo usuário, ou ainda características gerais do seu perfil, como a área de atuação. Esta abordagem pode não atender as expectativas do usuário, uma vez que não leva em consideração os seus interesses de aprendizagem atuais. Isto tem levado os pesquisadores a estudarem abordagens de recomendação de OAs sensíveis ao contexto [8].

Com base no exposto, este artigo propõe um modelo para sistemas de recomendação de OAs denominado REBASS (REcomendação BAseada em Similaridade de
Sessões), que considera a similaridade entre a sequência de objetos consultados durante a sessão atual do usuário com as sequências armazenadas no histórico de sessões do repositório.

Este modelo trata a recomendação de OAs como uma aplicação sensível ao contexto [9]. Um contexto é interpretado, conforme a definição de Dey, Abowd e Salber [10], como um conjunto de informações que descrevem a situação de uma entidade. No caso específico dos OAs, cada objeto acessado por um aluno durante uma atividade em aula ou pesquisa individual constitui o contexto daquele aluno em um momento específico.

Diferentes técnicas têm sido propostas para a avaliação de similaridade entre dois objetos, tais como: Levenshtein Distance, Sequence Alignment Distance, Hausdorff Distance, Lp Distance e a distância do Cosseno. Estas técnicas vêm sendo aplicadas em domínios como o reconhecimento de fala, análise de séries temporais, predição, mineração de dados, entre outras. Em geral as técnicas avaliam atributos dos objetos para calcular um valor numérico que indique a similaridade entre eles.

No caso das séries temporais, que são conjuntos de observações de eventos ordenados no tempo [11], podem ser aplicadas tanto técnicas que avaliem individualmente cada ponto das séries como também técnicas como a Dynamic Time Warping (DTW), que calcula uma série temporal média entre duas séries [12].

O REBASS trata a sequência de OAs consultados como uma série temporal, sendo aplicada a técnica DTW para encontrar sessões similares. A principal contribuição do modelo consiste no uso da sequência de acesso a OAs que está sendo criada na sessão corrente do usuário, permitindo assim uma recomendação contextualizada no interesse momentâneo do aluno. Um protótipo foi implementado e aplicado em uma avaliação envolvendo uma base real de sessões de acesso a OAs, obtida no sistema de EAD do curso de Sistemas de Informação das Faculdades Integradas de Taquara (FIT/FACCAT). O REBASS mostrou ser capaz de realizar recomendações adequadas de OAs, considerando o interesse corrente do aluno e sem a necessidade de existência de vínculos no repositório entre os objetos.

Este artigo está dividido em 9 seções. A seção 2 introduz o conceito de contexto e de aplicações sensíveis ao contexto adotado nessa pesquisa. A seção 3 apresenta uma introdução às séries temporais. A seção 4 ilustra algumas técnicas de análise de similaridade, especialmente a Dynamic Time Warping, adotada pelo modelo. Na seção 5 são descritos os trabalhos relacionados. A seção 6 apresenta o modelo do REBASS. A seção 7 descreve os experimentos realizados. A seção 8 apresenta os resultados obtidos e sugere linhas de trabalhos futuros. Por fim, na seção 9 são apresentados os agradecimentos finais. 


\section{Aplicações Sensíveis ao Contexto}

Para Brown, Bovey e Chen [13] aplicações sensíveis ao contexto são aquelas cujo funcionamento é orientado principalmente pelo contexto atual do usuário. Estas podem ser divididas basicamente em contínuas e discretas. Nas aplicações contínuas as informações apresentadas ao usuário são atualizadas constantemente. Os autores citam como exemplo, um sistema de localização que continuamente exibe ao usuário uma seta indicando a direção que ele deve tomar para chegar a um determinado destino. A direção apontada pela seta varia conforme o usuário se movimenta. Em aplicações discretas, conjuntos independentes de informações estão associados a diferentes contextos, e são exibidos sempre que o usuário entra em um novo contexto. Segundo os autores a maioria das aplicações se enquadram melhor no conceito discreto.

Para Schilit e Theimer [14] o contexto é constituído por dados sobre a localização, identidade de pessoas e objetos próximos, mais as alterações nestes objetos. Em Brown, Bovey e Chen [13] são acrescentados ainda dados ambientais como a hora do dia, estação do ano e temperatura. O estudo de Pascoe, Ryan e Morse [15] sobre quais seriam as necessidades de pesquisadores de campo, como arqueólogos e biólogos, na utilização de PDA's considera que o contexto deve ter informações sobre identidade, localização, ambiente e tempo, já que para estes autores as atividades do usuário estão intimamente ligadas ao contexto no qual ele se encontra. $\mathrm{Na}$ definição de Dey, Abowd e Wood [16], o contexto é qualquer informação que possa ser usada para melhorar a experiência do usuário, o que implica em informações como o estado emocional do usuário, sua localização física, o ambiente social, objetos e pessoas próximas, entre outras.

Segundo Dey, Abowd e Salber [10], os trabalhos citados definem o conceito de contexto através de exemplos de aplicações. O que os torna específicos para determinados domínios, dificultando sua aplicação em outras áreas já que não deixam claro como saber se um novo tipo de informação pode ser classificado como contexto. Por outro lado, segundo os autores, definições mais genéricas como as propostas por Brown [17], Franklin e Flashbart [18], Ward, Jones e Hooper[19] e Rodden et al. [20] acabam sendo difíceis de serem implementadas pois não determinam como identificar os elementos que constituem um contexto.

Para Schilit, Adams e Want [21], os aspectos mais importantes de um contexto são: aonde o usuário está, com quem o usuário está e quais recursos estão próximos. Os autores ainda dividem o ambiente de execução em Ambiente Computacional, como processadores disponíveis, dispositivos acessíveis, rede, conectividade e custos computacionais; Ambiente do Usuário, como localização, pessoas próximas e situação social; Ambiente Físico, como luminosidade e nível de ruído.

Neste artigo, será adotada a seguinte definição de contexto:

"Contexto é qualquer informação que possa ser usada para caracterizar a situação de entidades que são consideradas relevantes para a interação entre um usuário e uma aplicação, incluindo o próprio usuário e a aplicação. Contextos são, tipicamente: a localização, identidade e o estado de pessoas, grupos e objetos físicos e computacionais" [10].

Nesta definição, o objetivo de conhecer o contexto é determinar qual atividade o usuário está tentando realizar. Já que é difícil determinar diretamente quais são os objetivos do usuário, dados do contexto podem ajudar a inferir esta informação.

Outra questão levantada por Dey, Abowd e Salber [10], é a da diversidade de informações que podem compor o contexto. Para lidar com esta diversidade, é recomendável que se busque uma forma de categorização dos dados contextuais, para facilitar sua compreensão. Os autores propõem uma classificação simples destas informações, baseada nas entidades envolvidas no contexto avaliado. As entidades mais importantes identificadas pelos autores são: lugares, pessoas e coisas. Lugares são regiões de espaço geográfico como salas e construções. Pessoas podem ser tanto indivíduos como grupos distribuídos ou compartilhando a mesma região. Coisas podem tanto ser objetos físicos como componentes de software ou artefatos.

Também são definidas quatro categorias essenciais da informação de contexto: Identidade, localização, estado ou atividade e tempo.

Identidade refere-se a capacidade de atribuir um identificador único a cada entidade. Localização é mais do que simplesmente a posição atual da entidade. Pode abranger também dados como a orientação e elevação, bem como informações que permitam deduzir as relações espaciais entre as entidades. Por exemplo, a possibilidade de determinar se um objeto está acima ou abaixo de outro. O conceito também se aplica a lugares e podem ter coordenadas relativas e absolutas.

Situação ou atividade refere-se a características inerentes à entidade vinculada ao contexto. Para lugares, podem ser informações como a temperatura, umidade, luminosidade ou nível de ruído. Para pessoas ou grupos pode significar o estado emocional, seus sinais vitais ou ainda a atividade na qual o usuário está envolvido. Para coisas, podem ser dados de sensores ou, no caso de softwares, podem ser atributos como o uso de CPU ou de memória. 
Por fim, o tempo identifica o momento em que os eventos ocorreram ou em que os dados contextuais tenham sido capturados, permitindo a análise histórica destes eventos. Assim como a localização, o tempo possui um significado mais amplo do que aparenta inicialmente. Ele pode representar um instante específico ou um intervalo, mas também ser simplesmente um valor sequencial indicando a ordem em que os eventos ou as coletas de dados do contexto ocorreram.

Como justificativa para a escolha destas categorias os autores argumentam que através dos dados fornecidos por elas é possível inferir informações adicionais, estendendo o conhecimento sobre a situação. Por exemplo, o endereço de um usuário poderia ser derivado a partir da identidade, ou ainda, a quantidade de pessoas em uma sala em determinado momento permitiria deduzir se uma reunião irá ou não ocorrer.

A capacidade de organizar e armazenar os dados contextuais vinculados a uma entidade cria o conceito de histórico de contextos, ou trilhas, que é discutido a seguir.

Talvez o primeiro trabalho em ciência da computação a apresentar o conceito de trilhas como uma sequência de itens visitados por um usuário seja o de Bush e Wang [22]. Os autores previram que os computadores seriam uma ferramenta poderosa para organizar o conhecimento humano. Em seu trabalho é proposta uma máquina, denominada Memex, que armazenaria uma grande quantidade de documentos no formato de microfilmes. O usuário selecionaria e consultaria estes documentos em um sistema que permitiria inserir anotações criando vínculos (links) arbitrários, gerando assim uma trilha de documentos semanticamente encadeados conforme a preferência do usuário. Esta provavelmente é a primeira descrição do hipertexto, um conceito que só se popularizou no final do século XX.

Para Driver e Clarke [23] uma trilha é, no nível mais geral, uma coleção de localizações acompanhadas de informações associadas e uma ordem recomendada de visitação. Os autores utilizam a metáfora da trilha para capturar as atividades diárias de um usuário e adaptar o funcionamento de aplicações móveis sensíveis ao contexto, considerando-se o histórico de atividades e localizações visitados pelo usuário. Aplicações sensíveis as trilhas devem processar um conjunto de informações contextuais, como condições do trânsito em diferentes rodovias, meios de transporte e condições do tempo, para oferecer serviços como, por exemplo, a sugestão de rotas para motoristas em cidades desconhecidas ou gerenciar a logística de serviços de entrega.

Spence, Driver e Clarke [24] estendem o trabalho anterior [23], ao considerar que é possível analisar também o histórico de trilhas recomendadas. Os autores chamam de trail histories as informações sobre como o usuário fez uso das trilhas sugeridas no passado. Isto inclui informações como a trilha inicial proposta pela aplicação, todas as reorganizações de trilhas que ocorreram, a trilha atual seguida pelo usuário e ainda outras informações do contexto, que podem ajudar a identificar por que o usuário não seguiu a trilha original.

Posteriormente, em Driver e Clarke [25], uma trilha é descrita como uma coleção de atividades a serem executadas pelo usuário, semelhante a uma 'to-do list' mas cuja sequência de execução é ordenada conforme o contexto e as preferências do usuário. Os autores argumentam que a trilha pode ser usada como um modelo genérico capaz de satisfazer os requisitos de aplicações de gerenciamento de tempo sensíveis ao contexto. As trilhas superam as limitações das técnicas tradicionais deste tipo de aplicação, geralmente baseada em listas estáticas de atividades, substituindo-as por listas reordenadas dinamicamente de acordo com o contexto do usuário. Partindo deste princípio, os autores apresentam um framework que visa oferecer funcionalidades para a geração de trilhas em resposta a alterações no contexto atual do usuário.

A análise destes dados pode auxiliar no desenvolvimento de aplicações de contextos colaborativos. Para os autores [25], o conceito de collaborative context refere-se ao compartilhamento de dados entre dispositivos em ambientes sensíveis ao contexto. Através da análise dos históricos de trilhas, algumas funcionalidades deste tipo de aplicação podem ser aperfeiçoadas, como por exemplo, impedir gerações desnecessárias de trilhas, a verificação de hipóteses e adaptação baseada no feedback do usuário.

\section{Séries Temporais}

Séries temporais são conjuntos de observações ordenadas cronologicamente [11]. Segundo Esling e Agon [26], em quase todas as áreas da ciência há a necessidade de se realizar medições de variáveis ao longo de um período de tempo, o que leva a criação destes conjuntos de dados ordenados.

Por exemplo na ciência econômica, segundo Hamilton [27], as séries temporais são utilizadas em econometria, já que boa parte da pesquisa nesta área consiste em modelar o comportamento de variáveis econômicas ao longo do tempo. Os modelos que descrevem as séries temporais em geral constituem-se de equações diferenciais, que o autor define como expressões que relacionam uma variável $\mathrm{y}_{\mathrm{t}}$ com seus valores prévios $\left(\mathrm{y}_{\mathrm{t}-1}, \mathrm{y}_{\mathrm{t}-2}, \ldots, \mathrm{y}_{\mathrm{t}-\mathrm{n}}\right)$.

Um exemplo é função de Goldfelds's, que estima a demanda de dinheiro pelos Estados Unidos: $m_{\mathrm{t}}=0.27+$ $0.72 m_{t}-1+0.19 I_{t}-0.045 r_{b t}-0.019 r_{c t}$. 
O modelo de Goldfeld's relaciona a quantidade de dinheiro real em poder do público $\left(m_{t}\right)$ com os rendimentos reais globais $\left(I_{t}\right)$, as taxas de juros em contas bancárias $\left(r_{b t}\right)$ e as taxas de juros em papéis comerciais $\left(r_{c t}\right)$. Percebe-se que o valor da variável $m$ em um momento $t$ depende parcialmente do valor da variável $m$ no momento anterior $\left(m_{t-1}\right)$. Esta é uma equação diferencial de primeira ordem, já que somente o primeiro período anterior $(t-1)$ é utilizado na equação [27].

Uma série temporal é denominada como Determinística quando puder ser descrita através de uma função como a de Goldfeld's. Caso haja um componente aleatório $\varepsilon$ nesta função $\left(\mathrm{y}_{\mathrm{t}}=\mathrm{f}(t, \varepsilon)\right)$, a série é denominada de Estocástica [28]. Além destas definições, Aiube [29] ainda classifica as séries temporais em:

A Contínuas: quando o conjunto for infinito não enumerável;

A Multivariada: quando a série for representada por um vetor;

A Discretas: quando o conjunto de dados for finito ou infinito enumerável;

A Multidimensional: quando $t$ assume um valor maior do que 1 .

Conforme Shikida e Margarido [30] uma série temporal possui geralmente os seguintes componentes:

A Ciclos: caracterizados por longas ondas, mais ou menos regulares, em torno de uma linha de tendência;

A Tendências: utilizadas para avaliar o comportamento de longo prazo das variáveis em análise. Pode ser determinística ou estocástica;

A Sazonalidade: utilizado para avaliar os padrões regulares da série, afetados por eventos periódicos como temperatura, safra ou entressafra, entre outros;

A Componente Aleatório: constitui-se de valores residuais que não podem ser explicados por um dos três componentes anteriores.

Considerando-se a definição de série temporal dada por Esling e Agon [26] e Fu [11], percebe-se que as sequências de OAs acessados por um usuário durante uma sessão de consulta a um repositório podem ser descritas como uma série temporal, desde que estejam cronologicamente ordenadas e os aspectos relevantes para a análise de similaridade entre as sequências puderem ser modelados com funções matemáticas. A Figura 1 ilustra esta correlação. Considerando uma sequência de contextos como uma série de observações feitas ao longo do tempo, referentes aos contextos visitados por uma entidade, conclui-se que estas sequências podem ser tratadas como séries temporais artificiais.

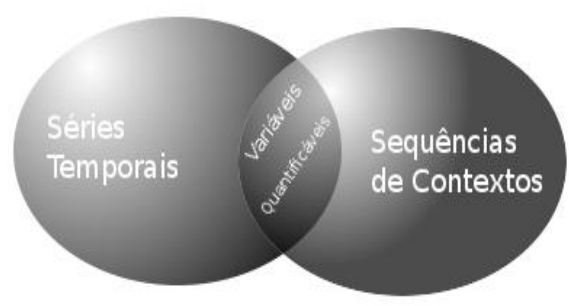

Figura 1: Sequências de Contextos como Séries Temporais

\section{Análise de Similaridade}

Segundo Cha [31], do ponto de vista matemático e científico a distância ou dissimilaridade é um valor que expressa o quão distante dois objetos estão. Medidas quantitativas são em geral chamadas de métricas, enquanto outras medidas não-métricas são as vezes chamadas de divergências. Já a similaridade ou proximidade entre objetos é, em alguns trabalhos, chamada de coeficiente de similaridade.

Já na área de descoberta de conhecimento em bases de dados a tarefa de clusterização é a que possui uma relação mais próxima com o conceito de similaridade. A clusterização, ou agrupamento, tem como objetivo particionar os registros de uma base de dados em subconjuntos chamados clusters, de tal forma que os elementos que compõem um cluster compartilhem um conjunto de propriedades que permitam distingui-los dos demais clusters. O resultado ótimo desta tarefa consiste em obter a maior similaridade intra-cluster e a maior distância inter-cluster [32].

Os algoritmos de clusterização necessitam trabalhar sobre estruturas de dados capazes de armazenar os objetos a serem processados ou as informações sobre eles. Existem duas estruturas que normalmente são utilizadas para representar os dados a serem clusterizados, a Matriz de Dados e a Matriz de Similaridade [32].

$$
X=\left[\begin{array}{ccccc}
x_{11} & x_{12} & x_{13} & \ldots & x_{1 \mathrm{p}} \\
x_{21} & x_{22} & x_{23} & \ldots & x_{2 \mathrm{p}} \\
x_{31} & x_{32} & x_{33} & \ldots & x_{3 \mathrm{p}} \\
\vdots & \vdots & \vdots & \ddots & \vdots \\
x_{n 1} & x_{n 2} & x_{n 3} & \ldots & x_{n p}
\end{array} \mid\right.
$$

Figura 2: Matriz de Dados [32] 
A Matriz de Dados $x=n \times p$, ilustrada na Figura 2 , é construída a partir da lista de objetos a serem agrupados. Cada linha $1 . . . n$ representa um objeto e cada coluna $1 \ldots p$ o valor de um atributo do objeto no espaço $p$ dimensional.

A Matriz de Similaridade, ilustrada na Figura 3, é construída a partir da distância entre pares de objetos. A função $d_{(i, j)}$ representa a medida de distância ou similaridade entre um objeto $i$ e um objeto $j$. Quanto mais próximo de 0 (zero) for a distância entre dois objetos, mais similares eles serão.

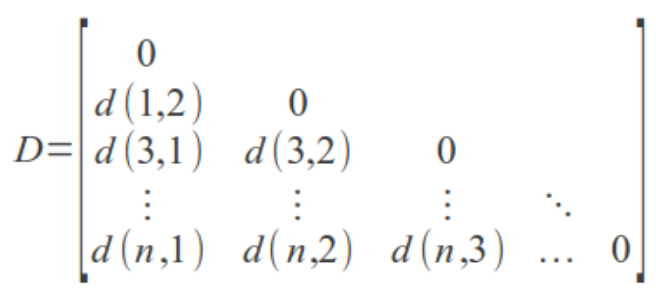

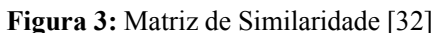

Quando se trata de analisar a similaridade entre duas sequências a tarefa se torna mais complexa, pois é necessário avaliar não apenas os atributos dos objetos individualmente, mas também a ordem na qual os objetos aparecem na sequência. Alguns trabalhos, como Abraham e Lal [33] e García-Díez et al. [34], denominam este problema como "Alinhamento de Sequências" e apresentam técnicas para o tratamento de problemas específicos, que levam em consideração as sequências de objetos a serem comparados.

Skopal e Bustos [35] descrevem algumas técnicas de análise de similaridade de uso geral que podem ser aplicadas a este domínio. A Edit Distance, também conhecida como distância de Levenshtein mede a quantidade mínima de operações de edição necessárias para transformar uma string em outra. Nessa técnica são permitidas operações de inserção, exclusão e substituição.

$\mathrm{Na}$ Longest Common Subsequence (LCS) uma sequência $x$ é considerada uma subsequência de uma sequência $y$ apenas se houver uma ordem estritamente crescente de índices que apontem para símbolos iguais, mas não necessariamente adjacentes, em ambas as sequências. Dadas duas sequências $x$ e $y$, uma terceira sequência $z$ é uma subsequência comum se for uma subsequência tanto de $x$ quanto de $y$. A subsequência de maior comprimento encontrada é chamada de LCS.

A Cosine Measure and Distance, dada pela equação 1 , mede o cosseno do ângulo entre dois vetores em situações onde a magnitude dos vetores é irrelevante. Esta é uma medida de similaridade que retorna 0 (zero) para vetores paralelos $\left(0^{\circ}\right)$ e 1 (um) para vetores ortogonais $\left(90^{\circ}\right)$. A partir da fórmula $1-S_{c o s}$ obtém-se uma medida da distância entre os vetores.

$$
S_{\cos }=\frac{\sum_{i=1}^{d} x_{i} y_{i}}{\sqrt{\sum_{i=1}^{d} x_{i}^{2} \sum_{i=1}^{d} y_{i}^{2}}}
$$

Outra técnica conhecida é a Dynamic Time Warping (DTW) [12], que é utilizada na análise de similaridade em séries temporais, como por exemplo a análise do histórico de preços de ações e o reconhecimento de fala.

A Figura 4 ilustra esta técnica. Considerando-se duas séries temporais $\mathrm{S}=\left\{s_{1}, s_{2}, \ldots, s_{i}, \ldots, s_{n}\right\}$ e $\mathrm{T}=\left\{t_{1}, t_{2}, \ldots, t_{j}, \ldots\right.$, $\left.t_{m}\right\}$, a técnica DTW alinha ambas as séries na forma de um grid $n \times m$, onde $n$ e $m$ são os tamanhos das respectivas séries e cada ponto $\{i, j\}$ do grid corresponde a um alinhamento entre os elementos das séries. $O$ valor de cada ponto é obtido utilizando-se alguma função de distância $\delta$ capaz de calcular a diferença entre dois pontos das séries.

Um exemplo de função de distância seria calcular a magnitude da diferença, dada por $\delta(i, j)=|i-j|$, ou o quadrado da diferença, dado por $\delta(i, j)=(i-j)^{2}$. Quando não houver diferença entre as séries, o warping path irá corresponder a linha diagonal.

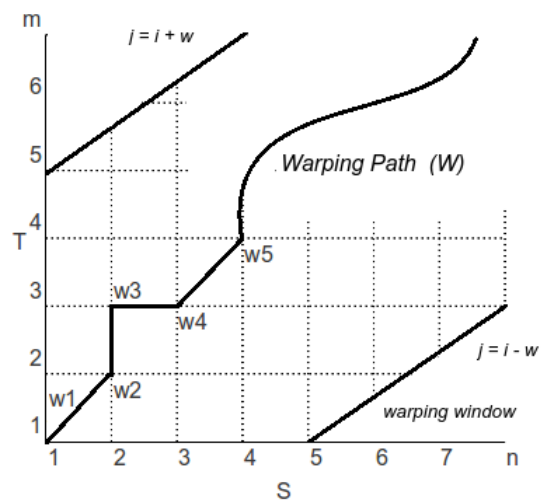

Figura 4: Dynamic Time Warping [12]

Tendo-se definido a função de distância entre pontos, o cálculo da distância entre as séries consiste em utilizar técnicas de programação dinâmica para encontrar o caminho $\mathrm{W}=\{\mathrm{w} 1, \mathrm{w} 2, \ldots, \mathrm{wk}\}$ através do grid que corresponda ao menor somatório de distâncias individuais. Para Berndt e Clifford [12], isto pode ser formalmente definido como um problema de minimização sobre o total de possíveis Warping Paths, formalizado conforme a equação 2 , onde $\delta$ é a função de distância no ponto $w_{k}$ do grid e $p$ é o último ponto de comparação entre as séries. 


$$
\operatorname{DTW}_{(S, T)}=\min _{w}\left[\sum_{k=1}^{p} \delta\left(w_{k}\right)\right]
$$

Para a resolução deste tipo de problema é necessário o uso de uma variável stage, variáveis de estado e variáveis de decisão. No caso da DTW, a variável stage impõe uma ordem monótona aos eventos representados pela série e é dada pela dimensão TEMPO das séries. As variáveis de estado são os pontos individuais do grid. As variáveis de decisão, que definem as transições de estados possíveis, correspondem às restrições que definem quais os caminhos são possíveis de serem percorridos de forma a reduzir o tamanho do espaço de soluções, chamado de warping window.

A resolução do problema de minimização é obtida através da relação recursiva formalizada na equação 3 , que calcula a distância acumulativa para cada ponto do grid que esteja dentro da warping window. A distância acumulativa é dada pela soma da distância entre o ponto atual e a menor distância acumulativa dos seus pontos vizinhos. Este é um algoritmo simétrico, uma vez que ambos os pontos diagonais predecessores, $(i-1, j)$ e $(i, j$ - 1), são utilizados.

$$
\gamma(i, j)=\delta(i, j)+\min [\gamma(i-1, j), \gamma(i-1, j-1), \gamma(i, j-1)]
$$

\section{Trabalhos Relacionados}

Zapata et al. [36] apresentam um framework denominado DELPHOS para auxiliar os usuários na realização de consultas personalizadas em repositórios. $\mathrm{O}$ framework adota uma abordagem híbrida, que combina as abordagens Colaborativa, ao analisar o histórico dos OAs mais acessados e a avaliação dos usuários, Baseada em Conteúdo, ao calcular o grau de similaridade entre dois OAs, e Demográfica ao calcular a similaridade entre usuários.

Cazella et al. [37] propõem um modelo denominado RECoaComp (REComendador de objetos de aprendizagem baseado em Competências), que tem como objetivo filtrar os OAs de um repositório de acordo com as competências a construir, sinalizadas no perfil do usuário. Neste modelo, o professor primeiramente seleciona os objetos que deseja utilizar, identificando a lista de competências que cada OA ajuda a desenvolver. Em seguida, os alunos realizam uma auto-avaliação em relação as competências identificadas pelo professor. Finalmente, o sistema sugere os OAs de acordo com as competências de cada aluno. Este modelo também calcula e armazena a lista de usuários similares para cada usuário. Para o sis- tema de recomendação, alunos similares significam alunos que costumam avaliar o conteúdo dos objetos de maneira semelhante. Este aspecto permite prever se um aluno deve receber a recomendação de um determinado OA ou não.

Rocha et al. [38] desenvolveram um módulo de recomendação de OAs integrado ao ambiente virtual AMADEUS (http://amadeus.cin.ufpe.br/). O algoritmo de recomendação apresentado neste trabalho representa os metadados dos OAs na forma de vetores, onde os termos presentes em cada descrição correspondem às dimensões do vetor, e a frequência de termos corresponde ao valor de cada dimensão. Sobre esta representação é aplicada a técnica $k$-nearest neighbors para filtrar os objetos a serem recomendados.

Os trabalhos relacionados utilizam como base para a análise de similaridade o conjunto total de características dos objetos ou o histórico completo de objetos acessados pelo usuário. Esta técnica pode gerar recomendações que não atendam as necessidades do usuário em relação aos seus interesses atuais.

Para casos como este alguns trabalhos propõem uma abordagem para recomendação de OAs sensível ao contexto. Por exemplo, Silva et al. [8] propõem um ambiente de recomendação de OAs baseado em um modelo multiagente e voltado para dispositivos móveis, considerando os recursos limitados existentes nestes dispositivos.

O modelo proposto por Silva et al. [8] baseia-se em um componente que os autores denominam "Ontologia de Contexto Estático", que armazena os locais e horários de preferência do estudante, adotando desta forma, as preferências recentes do estudante para determinar o seu contexto.

A principal diferença entre a proposta de Silva et al. e o REBASS é o período de tempo considerado para determinar o contexto do aluno. O REBASS restringe a interpretação dos interesses do aluno somente ao histórico da sessão atual de acesso ao ambiente de EAD, por considerar que esta abordagem levaria a uma identificação mais precisa destes interesses, gerando assim melhores recomendações. Considera-se que o histórico de uma sessão irá conter uma lista de OAs relacionados ao tema específico de pesquisa de um estudante em determinado momento, ao passo em que o histórico completo do estudante conteria OAs relativos a várias disciplinas e temas de pesquisa.

Outra questão a ser analisada é quanto ao desempenho das técnicas apresentadas. Nunes e Cazella [39], por exemplo, afirmam que a Filtragem Colaborativa possui um desempenho $\Omega\left(\mathrm{n}^{2}\right)$, e mesmo a recomendação Baseada em Conteúdo pode tornar-se inviável em função da quantidade de OAs disponíveis no repositório, ou exigir 
um cálculo prévio da similaridade entre os OAs, antes que qualquer recomendação possa ser feita.

Tendo em vista estas questões, o modelo proposto utiliza como base para análise de similaridade apenas a sequência de OAs acessados durante a sessão atual do usuário. Esta sequência é então comparada com outras sessões armazenadas no histórico do repositório, visando identificar aquelas mais semelhantes em relação a sessão atual. A recomendação é feita selecionando-se, nas sessões similares, aqueles OAs que ainda não tenham sido acessados durante a sessão atual. Este modelo possui as seguintes características:

(i) a sequência de OAs da sessão atual identifica, de forma implícita, qual o interesse do usuário no momento, o que permite uma recomendação mais precisa em relação ao contexto atual do usuário; (ii) a sessão atual pode trazer, também de forma implícita, o contexto atual do usuário, como por exemplo, qual curso ou disciplina ele está cursando no momento, quem são seus colegas de classe, entre outras informações inferíveis;

(iii) a quantidade de OAs consultados em uma sessão normalmente é pequena, o que melhora o desempenho da análise de similaridade;

(iv) aplica uma técnica simplificada que pode ser facilmente adaptada a modelos mais complexos.

A principal contribuição desta pesquisa consiste no conceito de utilização da análise de similaridade para realizar a recomendação de OAs levando-se em consideração o interesse e o contexto atuais de cada estudante. Conforme demonstrado no experimento descrito na seção 7, comparando-se a sequencia de OAs consultados durante a sessão atual de consulta ao repositório com o histórico de sessões, é possível identificar sequências de OAs semelhantes, o que indica a possibilidade de que os interesses de pesquisa do usuário da sessão encontrada no histórico sejam similares aos interesses do usuário da sessão corrente. Com isto, têm-se um critério para a recomendação de objetos potencialmente úteis ao estudante, sem que seja necessário haver no repositório uma relação explícita entre os objetos.

\section{O Modelo REBASS}

O modelo proposto utiliza a técnica conhecida como DTW para analisar a similaridade da sessão do usuário atual e o histórico de sessões armazenados no repositório. Cada sessão é composta por uma sequência de OAs consultados pelo usuário, que é tratada pelo modelo como uma série temporal artificial [40]. Essa interpretação determinou a escolha da DTW como técnica de análise de similaridade, por ter sido desenvolvida especificamente para a análise de séries temporais. A arquitetura do modelo é composta por cinco camadas, ilustradas na Figura 5.

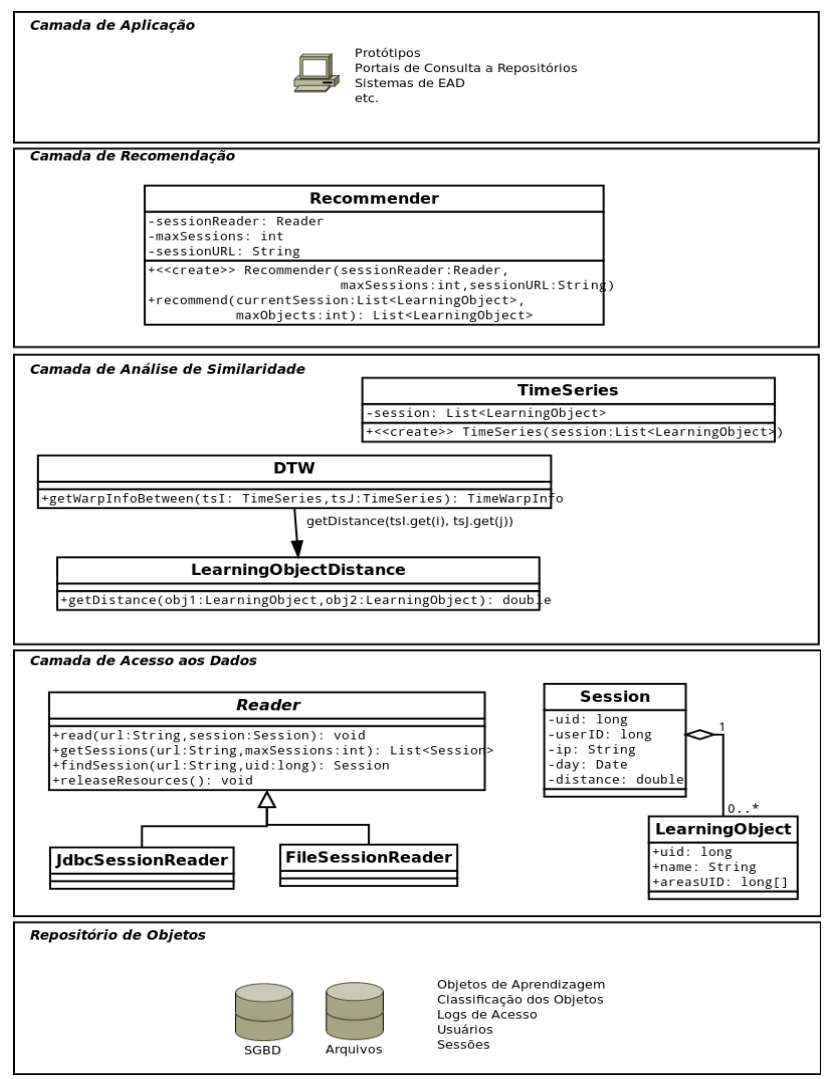

Figura 5: Arquitetura do REBASS

A primeira camada corresponde à aplicação que implementará o modelo como, por exemplo, um módulo no portal web de um repositório. A segunda camada é responsável por realizar a recomendação de OAs para uma sessão. A aplicação cliente utiliza o componente Recommender especificando qual componente da camada de acesso aos dados será utilizado para carregar os dados das sessões, qual a quantidade máxima de sessões que deverão ser analisadas, e a localização do repositório de objetos. Em seguida é feita uma chamada ao método recommend passando a sequência de OAs da sessão atual e a quantidade de OAs que devem ser recomendados. A terceira camada é responsável por realizar a análise de similaridade entre a sessão atual e as sessões armazenadas no repositório. A quarta camada realiza o acesso ao repositório, abstraindo os dados em uma representação interna de OAs e sessões. Por fim, a quinta camada representa o armazenamento físico do repositório.

Inicialmente, a aplicação deve ser capaz de representar os OAs consultados durante a sessão atual do usuário utilizando as classes Session e LearningObject da camada de acesso aos dados. Opcionalmente, pode-se carregar os dados de uma sessão específica a partir do histórico de sessões utilizando-se uma das implementações.

O parâmetro url do método read é uma String contendo a localização do repositório. O conteúdo desta String 
depende da implementação da classe Reader utilizada, podendo ser o path completo de um arquivo ou a String jdbc para conexão a um banco de dados.

Em seguida, a aplicação deve utilizar a classe $R e$ commender da camada de recomendação para obter a lista de objetos a serem recomendados ao usuário. A classe deve ser inicializada passando-se uma instância da classe Reader, que irá acessar o repositório, a quantidade máxima de sessões que serão lidas do histórico e a $u r l$ de acesso ao repositório.

Por fim é realizada uma chamada ao método recommend, passando por parâmetro os objetos da sessão atual e a quantidade máxima de objetos a serem recomendados.

O processo de recomendação é realizado em duas etapas. Na primeira etapa são identificadas as sessões mais similares à sessão atual, utilizando-se a técnica DTW. Inicialmente são obtidas as sessões do repositório, limitadas pelo número máximo de sessões a serem analisadas, utilizando-se a classe Reader e a url do repositório especificadas.

Em seguida a sequência de OAs de cada sessão é lida e representada na forma de uma série temporal, bem como a sequência da sessão corrente, utilizando-se a classe TimeSeries. A classe $D T W$ é então utilizada para calcular o valor da distância entre a série da sessão corrente e cada série obtida do histórico. Este valor é armazenado no atributo distance da objeto Session relativo a sessão do histórico.

Finalmente, após calcular as distâncias das séries do histórico em relação à série corrente, as sequências são ordenadas em ordem crescente de distância e utilizadas na segunda etapa para obter a lista de objetos a serem recomendados.

$\mathrm{Na}$ segunda etapa a lista ordenada de sessões similares é percorrida e cada OA que não estiver presente na sequência de OAs consultados na sessão corrente é então selecionado para ser recomendado ao usuário. Este processo é repetido até que o número máximo de recomendações seja atingido ou até o final da lista de sessões.

A camada de análise de similaridade calcula a distância de cada sessão encontrada no repositório, em relação à sessão corrente, utilizando a técnica DTW. Inicialmente ambas as sequências de OAs são tratadas como séries temporais $\mathrm{S}$ e $\mathrm{T}$ e são dispostas ortogonalmente formando um grid $\mathrm{n} \times \mathrm{m}$, conforme descrito na seção 4 . Cada ponto do grid, dentro da warping window, é preenchido com o valor da função de distância entre OAs implementada. Ao final deste processo é aplicado o algoritmo padrão da técnica DTW, que consiste em uma busca de menor caminho entre os caminhos possíveis no grid.

Para a implementação da função de distância $\delta$ entre os OAs foi desenvolvida uma técnica que compara a área temática dos objetos, retornando um valor entre $(0 . .1)$ conforme a distância encontrada. Esta técnica baseia-se no trabalho de Abraham e Lal [33] e calcula a similaridade em uma hierarquia de áreas temáticas através da comparação binária entre os níveis, conforme a equação 4 .

$$
\delta_{O A}(x, y)=1-\frac{X \cap Y}{\text { maxLength }(X, Y)}
$$

Na equação 4, o par (x,y) representa os OAs a serem comparados e $(\mathrm{X}, \mathrm{Y})$ são as respectivas hierarquias da área temática de cada objeto. A tabela 1 exemplifica o cálculo da distância entre dois OAs obtidos a partir do Banco Internacional de Objetos Educacionais [3].

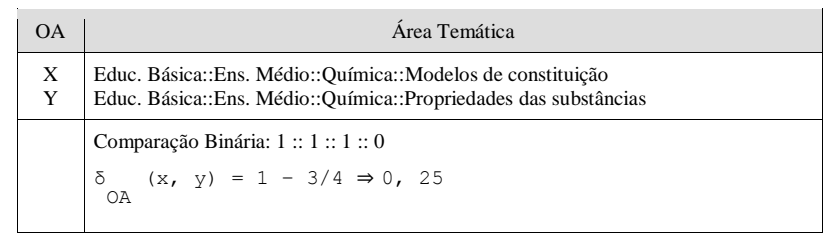

Tabela 1: Exemplo de cálculo de distância entre dois OAs

A função de distância implementada pela técnica consiste em uma comparação binária entre as hierarquias das áreas temáticas dos OAs, dividindo-se a quantidade de áreas iguais pelo tamanho da maior hierarquia. Uma característica desta técnica é que, assim como o trabalho de Abraham e Lal [33], o algoritmo continua calculando a comparação binária somente enquanto encontrar correspondência entre os níveis das duas hierarquias. O objetivo deste controle é reduzir a quantidade de falsos positivos durante a comparação. Por exemplo, se um objeto $\mathrm{X}$ está vinculado à área "Ciências::Física:Teoria Atômica" e um objeto Y está vinculado à área "Ciências::História da Ciência::Teoria Atômica", então a similaridade entre eles ocorre somente no topo da hierarquia (Ciências).

Por fim, caso os objetos sendo comparados possuam o mesmo identificador único (uid), significa que trata-se do mesmo objeto, e portanto pode-se considerar que o valor da distância é zero, sem a necessidade de calcular a similaridade entre as áreas temáticas. Esta função de distância é implementada pela classe LearningObjectDistance apresentada na Figura 6.

Uma vez definida a função de distância, é utilizada a técnica DTW para calcular a similaridade das sequências de OAs acessados durante a sessão atual em relação as sessões armazenadas nos históricos de uso do repositório. Pode-se optar por limitar a quantidade de sessões comparadas, considerando-se por exemplo, somente as sessões mais recentes, ou ainda as sessões de usuários com perfis mais similares aos do usuário atual. A técnica irá retornar as sessões mais similares à sessão atual com relação à sequência de OAs consultados. 


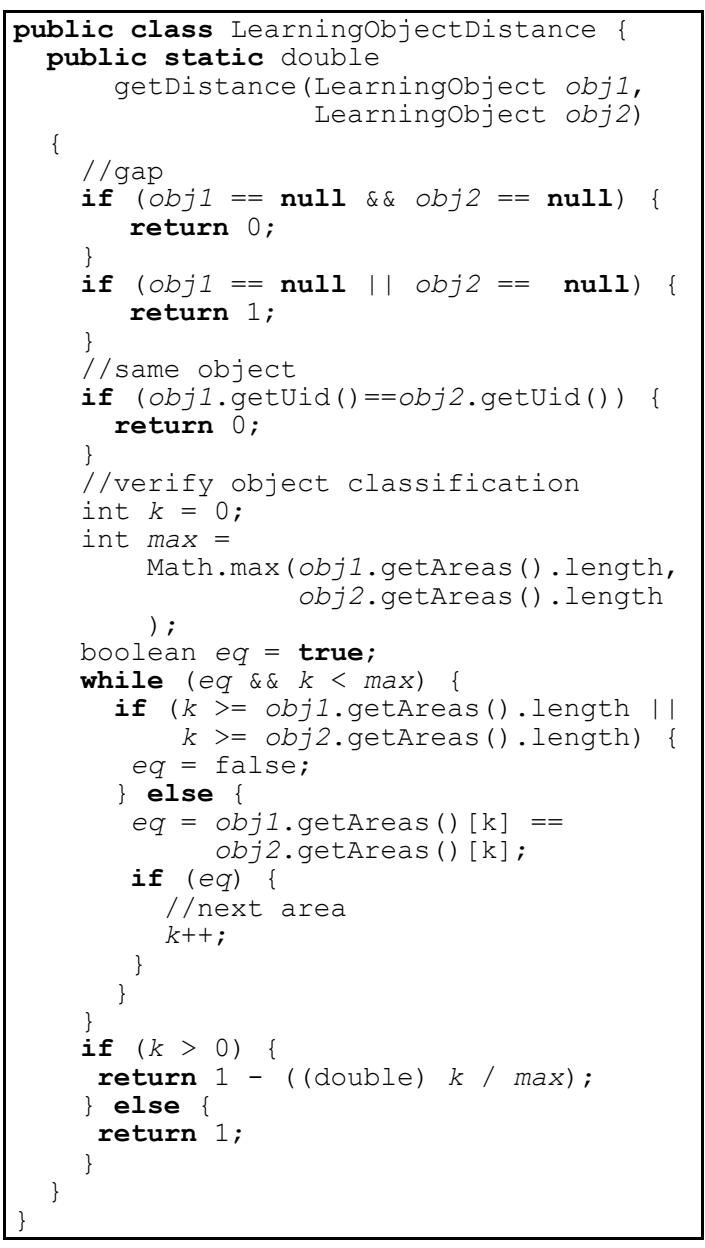

Figura 6: Implementação da função de distância entre dois OAs

A camada de recomendação então organiza as sessões obtidas em ordem crescente de distância, desconsiderando sessões cujo valor da distância seja igual a zero. A razão disto é que o valor zero indica uma sequência de OAs iguais aos da sessão atual, e portanto não haverá nestas sequências nenhum OA novo a ser recomendado. Por fim, o componente Recommender percorre a lista de sessões, selecionando os OAs que não constam na lista de OAs da sessão atual, até atingir o limite de OAs a serem recomendados, retornando-os à camada de aplicação.

\section{Protótipo e Avaliação}

Para a avaliação do modelo proposto foi implementado um protótipo desenvolvido em linguagem JAVA. O repositório foi armazenado em um banco de dados PostgreSQL.

Para implementação da técnica DTW foi utilizado o framework Open Source FastDTW, disponível em http://code.google.com/p/fastdtw/, que foi adaptado para utilizar a função de distância usada no REBASS. Os experimentos foram conduzidos em um Notebook DELL VostroTM com processador Intel Core ${ }^{\mathrm{TM}}$ i5 $2.40 \mathrm{GHz}$ com 6GB de memória RAM, e sistema operacional Ubuntu Linux 12.04 64-bits com kernel versão 3.2.0-51.

Os dados para realização do experimento foram obtidos a partir dos logs de acesso ao sistema Moodle (http://moodle.org) do curso de Sistemas de Informação das Faculdades Integradas de Taquara/RS (http://fit.faccat.br), referentes ao período de Agosto/2012 a Agosto/2013. Foram considerados os registros da tabela $m d l \_l o g$ referentes aos acessos de alunos aos materiais disponibilizados pelos professores. Estes materiais podem ser considerados exemplos de OAs [8] e são descritos na tabela $m d l$ resource a partir da qual foi obtida a descrição de cada objeto. Por fim, foi considerado como área temática de cada OA, o código de identificação do curso e do course module armazenados respectivamente nos campos course e cmid da tabela $m d l \_l o g$, criando assim uma hierarquia "Curso::Course_Module" para identificação da área temática de cada objeto.

A Figura 7 ilustra a estrutura dos dados utilizados para avaliação. A tabela $m d l \_l o g$ não possui originalmente um identificador da sessão do usuário. Para realizar o experimento, foi criada artificialmente uma tabela de sessões e um identificador de sessão. Para construção da tabela de sessões, os dados foram agrupados por Usuário (userid), IP e Data do acesso. Os campos "dataHora" e "dia" foram criados para auxiliar neste processo.

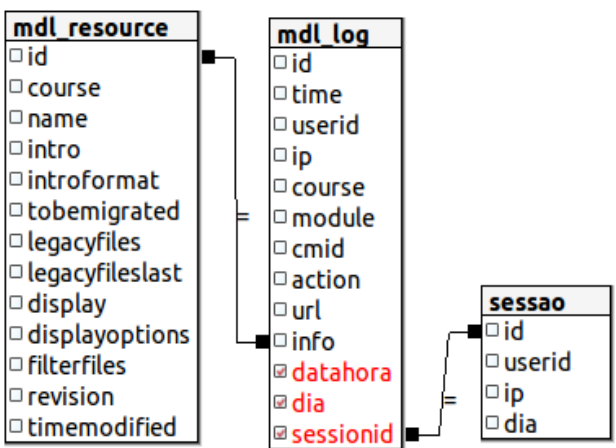

Figura 7: Tabelas do SGBD utilizadas na avaliação

Através deste critério, foram obtidas 11.039 sessões de usuários em 33.778 registros de acessos a OAs. A Figura 8 mostra a distribuição da quantidade de registros (eixo y) por sessão de usuário (eixo x). Percebe-se nesta Figura 8 que a maior sessão identificada possui 154 registros, porém a maioria das sessões possuem menos do que 25 registros. 


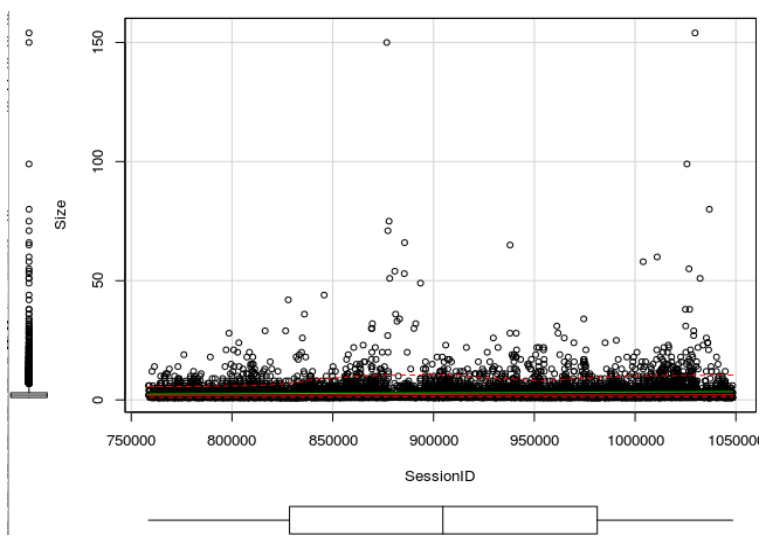

Figura 8: Gráfico de dispersão da quantidade de registros por sessão

O protótipo seleciona uma das sessões disponíveis no banco de dados e, a partir da sequência de OAs desta sessão, identifica as sessões similares e realiza a recomendação de novos objetos. O protótipo foi configurado para recomendar três OAs para a sessão selecionada. O resultado gerado pelo protótipo é um relatório do processamento indicando as sessões mais similares, os OAs recomendados e o tempo de processamento.

A biblioteca FastDTW implementa a técnica DTW para cálculo de distância entre duas séries temporais, representadas pela classe TimeSeries, que internamente utilizam vetores double[] para armazenar os valores numéricos que compõem cada série. Para que fosse possível utilizar esta biblioteca no cálculo de distância entre sequências de OAs algumas modificações foram necessárias.

Inicialmente, a classe TimeSeries recebeu um atributo session, composto por uma lista de OAs, e um construtor específico para este atributo. Em seguida, a classe $D T W$ foi adaptada para utilizar a função de distância LearningObjectDistance. Esta modificação envolveu alterar o método DynamicTimeWarp da classe para que utilizasse a função de distância de objetos na construção da matriz de distâncias, conforme ilustrado na Figura 9.

Após estas modificações, o protótipo foi capaz de utilizar a biblioteca FastDTW para calcular a distância entre duas sequências de contextos tratando-as como séries temporais e utilizando a técnica DTW. Os dados obtidos foram alimentados em um banco de dados PostgreSQL e a classe JdbcReader foi configurada para acessar este banco.

O protótipo recebe como parâmetro um número identificador de sessão que é carregado do banco de dados, simulando a sessão corrente de um usuário. Em seguida o processo de recomendação é ativado, realizando uma busca nas demais sessões presentes no repositório e recomendando os OAs presentes nas sessões mais simila-

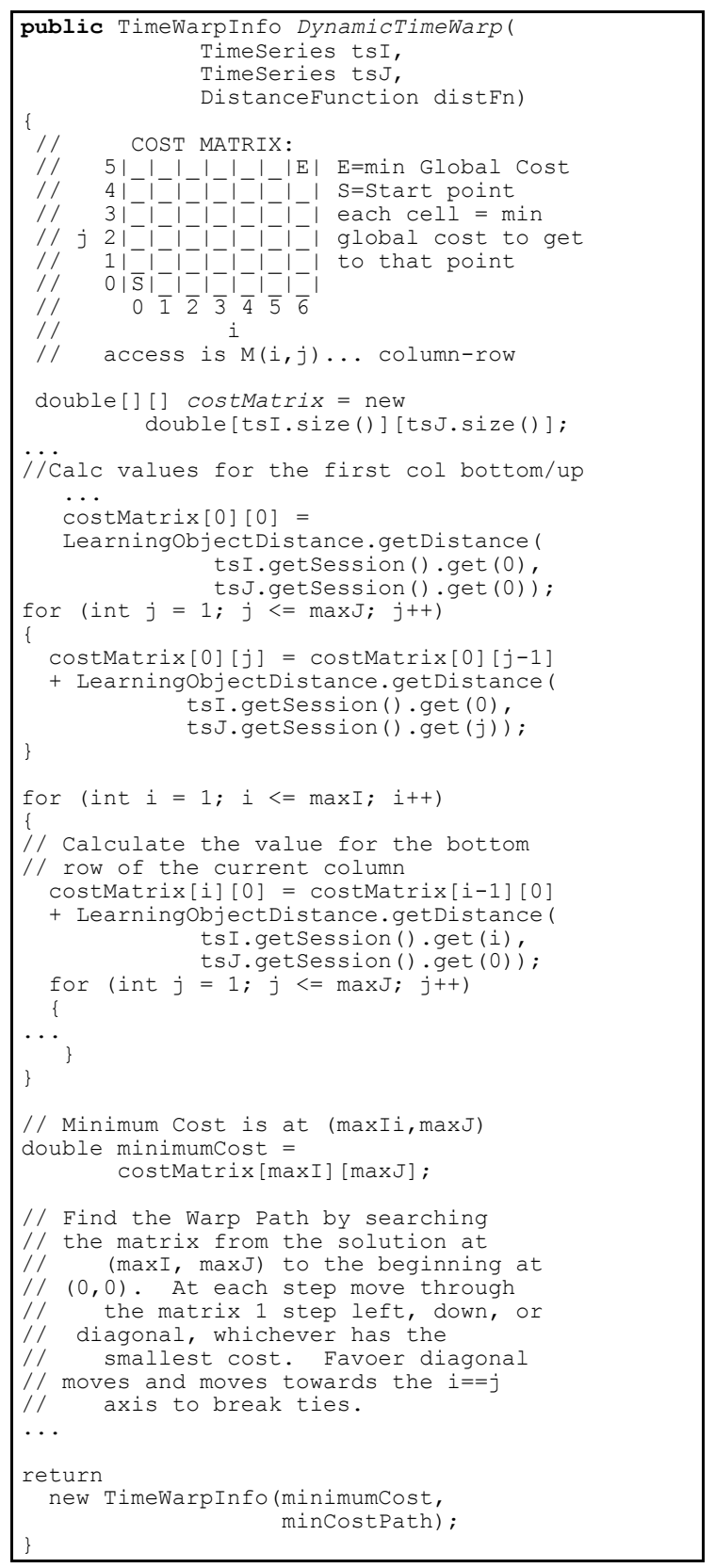

Figura 9: Método de cálculo de distância entre séries da classe DTW

res, que serão aquelas com o menor valor distância calculado pela FastDTW em relação à sessão atual.

A Figura 10 mostra o resultado da recomendação para a sessão de número [1044725], vinculada ao usuário de número [399]. Esta sessão possui quatro registros de acesso, referentes a três OAs identificados respectivamente pelos códigos [3183], [2761], e [290]. O componente de recomendação analisou 11.039 sessões, a uma taxa de 400 a 500 sessões por segundo, em cerca de 24 segundos. A sessão de número [1043715] foi a mais similar em relação a sessão corrente, com um valor de distância de 2.0 segundo a técnica DTW. Por fím, foram 


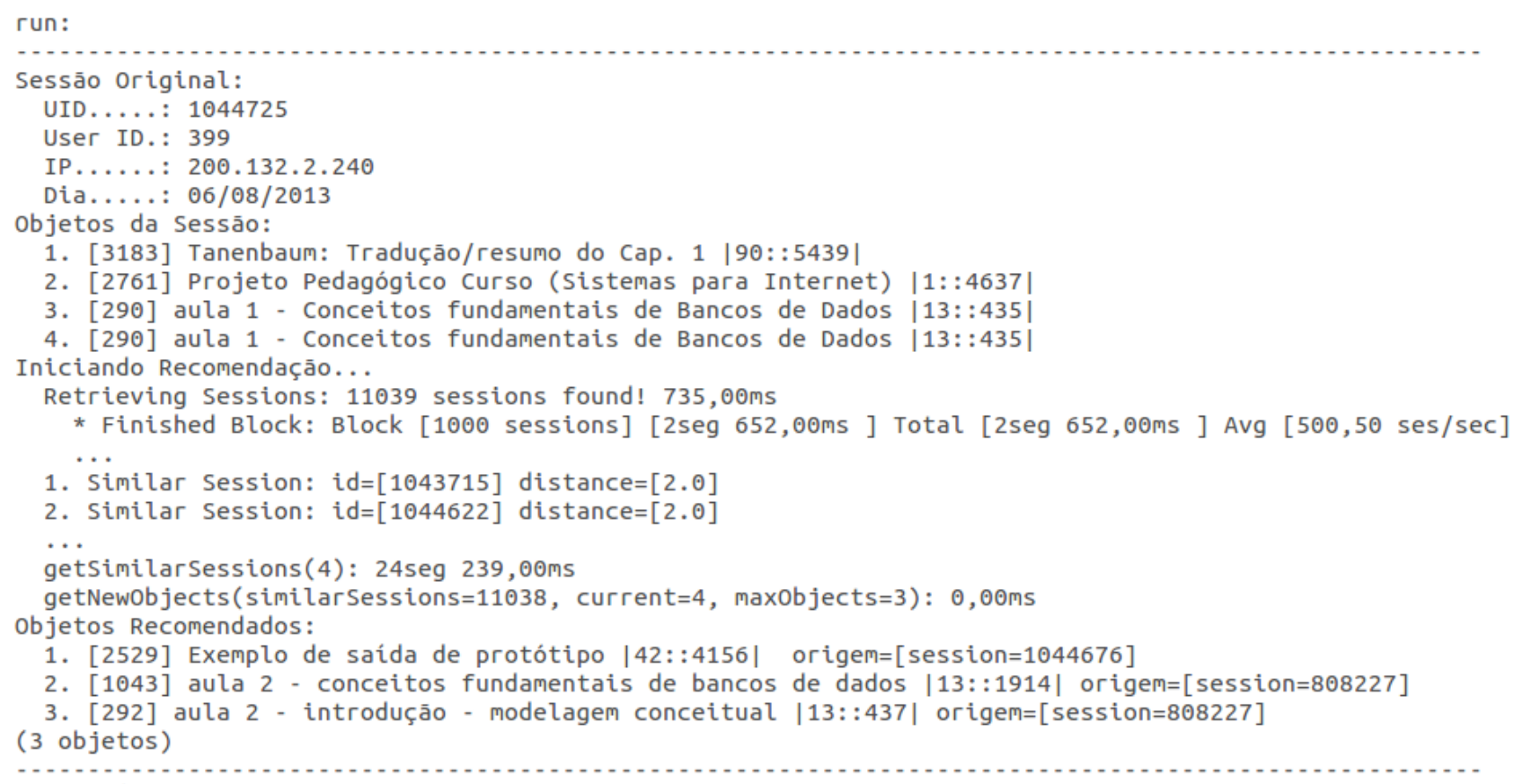

CONSTRUÍDO COM SUCESSO (tempo total: 24 segundos)

Figura 10: Resultado do processo de recomendação para uma sessão

recomendados os três OAs de números [2529], [1043] e [292].

Ao analisar o primeiro objeto recomendado, de número [2529] cuja descrição é "Exemplo de saída de protótipo" e cuja área temática é identificada por "42::4156", percebe-se que não é possível identificar semelhanças com nenhum dos objetos presentes na sessão atual do usuário. Porém ao analisar a sessão de origem desta recomendação, de número [1044676] e cujos registros são mostrados na Figura 11, pode-se verificar que a sequência de OAs é similar aos objetos da sessão [1044725]. É então possível deduzir que os interesses de aprendizagem do usuário durante a sessão [104467] eram similares aos interesses do usuário da sessão corrente. Partindo desta possibilidade, o protótipo então recomenda os OAs da sessão [1044676] que ainda não tenham sido consultados na sessão corrente.

Por fim, para avaliar a escalabilidade do modelo foi realizado um teste selecionando-se a maior sessão armazenada, com 154 registros de acesso. Conforme mostrado na Figura 11, a quantidade de sessões processadas por segundo não sofreu alteração significativa em função da quantidade de OAs da sessão atual, mantendo-se sempre uma média em torno de 500 sessões/segundo mesmo ao utilizar as maiores sessões disponíveis na base de dados.

\section{Conclusões e Trabalhos Futuros}

Este artigo apresentou um modelo para recomendação de OAs baseado na similaridade entre sequências de consultas realizadas em cada sessão de usuário. O modelo proposto utiliza a técnica denominada Dynamic Time Warping para calcular a similaridade entre a sequência de objetos consultados durante a sessão atual de um usuário e o conjunto de sessões armazenados nos $\log s$ de acesso à um repositório. Foi desenvolvida uma função para o cálculo da distância entre OAs, inspirada no trabalho de Abraham and Lal [33].

Foi desenvolvido um protótipo para avaliação do modelo proposto, utilizando o cenário real de acessos ao sistema de EAD do curso de Sistemas de Informação das Faculdades Integradas de Taquara (FIT/FACCAT). Conforme demonstrado na seção 7 , o modelo provou ser capaz de recomendar OAs potencialmente úteis ao interesse atual do usuário mesmo que os dados que descrevam os objetos não possuam nenhuma similaridade.

Como trabalhos futuros propõe-se o estudo da utilização de outras técnicas de análise de similaridade em sequências visando a comparação em relação a técnica DTW utilizada neste artigo, bem como o aperfeiçoamento da função de cálculo proposta para a distância entre objetos.

Além disso, seria relevante a realização de avaliações para uma melhor mensuração da eficácia do modelo proposto. Os modelos descritos nos trabalhos relacionados poderiam ser aplicados sobre os mesmos dados utilizados na avaliação do REBASS, permitindo uma comparação de eficácia entre os modelos. Em uma segunda etapa, o modelo proposto poderia ser implementado como um módulo experimental em uma aplicação, e então submetido a avaliação por um grupo de alunos em um ambiente 


\begin{tabular}{|c|c|c|c|c|c|c|c|}
\hline & $\begin{array}{l}\text { sessionid } \\
\text { bigint }\end{array}$ & $\begin{array}{l}\text { userid } \\
\text { bigint }\end{array}$ & $\begin{array}{l}\text { datahora } \\
\text { timestamp without time zone }\end{array}$ & $\begin{array}{l}\text { course } \\
\text { bigint }\end{array}$ & $\begin{array}{l}\text { cmid } \\
\text { bigint }\end{array}$ & $\begin{array}{l}\text { resource_uid } \\
\text { bigint }\end{array}$ & $\begin{array}{l}\text { resource_name } \\
\text { character varying(255) }\end{array}$ \\
\hline 1 & 1044676 & 1444 & $2013-08-0622: 30: 12$ & 42 & 4156 & 2529 & Exemplo de saída de protótipo \\
\hline 2 & 1044676 & 1444 & $2013-08-0622: 51: 15$ & 13 & 435 & 290 & aula 1 - Conceitos fundamentais de Bancos de Dados \\
\hline 3 & 1044676 & 1444 & $2013-08-0622: 51: 16$ & 13 & 435 & 290 & aula 1 - Conceitos fundamentais de Bancos de Dados \\
\hline
\end{tabular}

Figura 11: Objetos consultados durante a sessão 1044676

de EAD.

Por fim, a identificação de sessões similares poderá auxiliar em outras aplicações, como por exemplo a formação automática de grupos de estudos conforme o grau de similaridade entre os alunos, ou ainda a construção de perfis de estudantes conforme seu histórico de interesses.

\section{Agradecimentos}

Os autores agradecem à CAPES e ao $\mathrm{CNPq}$ pelo apoio ao desenvolvimento desta pesquisa. Os autores também agradecem ao Prof. Dr. Marcelo Azambuja, coordenador do curso de Sistemas de Informação das Faculdades Integradas de Taquara, e a Rodrigo Henrich, analista de suporte, pela disponibilização dos dados de acesso que permitiram a realização dos experimentos descritos neste artigo.

\section{Referências}

[1] D. A. Wiley. The instructional use of learning objects. http://reusability.org/read, Set. 2013.

[2] D. F. Audino, R. d. S. Nascimento. Objetos de aprendizagem - diálogos entre conceitos e uma nova proposição aplicada à educação. Revista Contemporânea de Educação, 5(10):128-148, 2012.

[3] Ministério da Educação. Banco Internacional de Objetos Educacionais. http://objetoseducacionais2.mec.gov.br/, Set. 2013.

[4] S. C. Cazella, E. B. Reategui, M. Machado, J. L. V. Barbosa. Recomendação de objetos de aprendizagem empregando filtragem colaborativa e competências. In Anais do Simpósio Brasileiro de Informática na Educação, Florianópolis, 2009

[5] R. Ghosh, M. Dekhil. Discovering user profiles. In Proceedings of the 18th international conference on World wide web, $W W W$ '09, New York, p. 1233-1234, 2009
[6] T. T. Primo, R. M. Vicari, J. M. C. da Silva. Rumo ao uso de metadados educacionais em sistemas de recomendação. In Anais do Simpósio Brasileiro de Informática na Educação, João Pessoa, 2010.

[7] H. S. Al-Khalifa. Building an arabic learning object repository with an ad hoc recommendation engine. In Proceedings of the 10th International Conference on Information Integration and Webbased Applications \& Services, iiWAS '08, New York, p. 390-394, 2008

[8] L. C. N., da Silva, F. M. Mendes Neto, L. Jácome Júnior. Mobile: Um ambiente multiagente de aprendizagem movel para apoiar a recomendacao sensivel ao contexto de objetos de aprendizagem. In Anais do Simpósio Brasileiro de Informática na Educação, Aracajú, 1(1), 2011

[9] G. D. Abowd, A. K. Dey, P. J. Brown, N. Davies, M. Smith, P. Steggles. Towards a better understanding of context and context-awareness. Handheld and ubiquitous computing, p. 304-307, 1999

[10] A. K Dey, G. D. Abowd, D. Salber. A Conceptual Framework and a Toolkit for Supporting the Rapid Prototyping of Context-Aware Applications. Human-Computer Interaction, 16(2): 97166, 2001.

[11] T. C. Fu. A review on time series data mining. Engineering Applications of Artificial Intelligence, 24(1):164-181, 2011.

[12] D. Berndt, J. Clifford. Using dynamic time warping to find patterns in time series. In Proceedings of the AAAI-94 Workshop on Knowledge Discovery in Databases, p. 229-248, 1994.

[13] P. J. Brown, J. D. Bovey, X. Chen. Context-aware applications: from the laboratory to the marketplace. IEEE Personal Communications, 4(5):5864, 1997.

[14] B. N. Schilit, M. M. Theimer. Disseminating active map information to mobile hosts. IEEE 
Network, 8(5):22-32, 1994.

[15] J. Pascoe, N. S. Ryan, D. R. Morse. Human Computer Giraffe Interaction: HCI in the Field. In Workshop on human computer interaction with mobile devices, 1998.

[16] A. K. Dey, G. D. Abowd, A. Wood.. CyberDesk: a framework for providing self-integrating context-aware services. In Proceedings of the 3rd international conference on intelligent user interfaces, IUI '98, New York, p. 47-54, 1998.

[17] P. J. Brown. The Stick-e document: a framework for creating context-aware applications. In Proceedings of Eletroninc Publishing, IFIP 96, Laxenburg, Austria, 1996.

[18] D. Franklin, J. Flaschbart. All gadget and no representation makes jack a dull environment. In Proceedings of Spring Symposium on Intelligent Environments, AAAI 98, Menlo Park, 1998.

[19] A. Ward, A. Jones, A. Hooper. A new location technique for the active office. IEEE Personal Communications, p. 42-47, 1997.

[20] T. Rodden, K. Cheverst, K. Davies, A. Dix. Exploiting context in HCI design for mobile systems. In Workshop on Human Computer Interaction with Mobile Devices, Glasgow, 1998.

[21] B. Schilit, N. Adams, R. Want. Context-aware computing applications. In Workshop on Mobile Computing Systems and Applications, p.85-90, 1994

[22] V. Bush, J. Wang. As we may think. Atlantic Monthly, 176, p. 101-108, 1945.

[23] C. Driver, S. Clarke. Context-aware trails [mobile computing]. Computer, 37(8):97-99, 2004.

[24] M. Spence, C. Driver, S. Clarke. Sharing Context History in Mobile, Context-Aware Trails-Based Applications. In anais do ECHISE 2005, Munich, 2005.

[25] C. Driver, S. Clarke. An application framework for mobile, context-aware trails. Pervasive Mob. Comput., Amsterdam, 4(5):719-736, 2008.

[26] P. Esling, C. Agon. Time-series data mining. ACM Comput. Surv., New York, 45(1):12:1-
12:34, 2012.

[27] J. Hamilton. The Time Series Analysis. Princeton University Press, Princeton, 1994.

[28] R. L. S. Bueno, Econometria de Séries Temporais. Cengage Learning, São Paulo, 2008.

[29] F. A. L. Aiube. Econometria para séries financeiras. Working Paper, Pontifícia Universidade Católica do Rio de Janeiro, 2007.

Disponível em http://www.ind.pucrio.br/UserFiles/File/aiube/Econometria\%20Serie s\%20Financeiras.pdf.

[30] P. F. A. Shikida, M. A. Margarido. Uma análise econométrica de sazonalidade dos preços da canade-açúcar, estado do Paraná, 2001-2007. Informações Econômicas, São Paulo, v. 39 p. 13, 2009.

[31] S. H. Cha. Comprehensive Survey on Distance / Similarity Measures between Probability Density Functions. International Journal of Mathematical Models and Methods in Applied Sciences, 1(4):300-307, 2007.

[32] R. Goldschmidt, E. Passos. Data mining: um guia prático. Elsevier, Rio de Janeiro, 2005.

[33] S. Abraham, P. S. Lal. Spatio-temporal similarity of network-constrained moving object trajectories using sequence alignment of travel locations. Transportation Research Part C: Emerging Technologies, 23(0):109-123, 2012.

[34] S. García-Díez, F. Fouss, M. Shimbo, M. Saerens. A sum-over-paths extension of edit distances accounting for all sequence alignments. Pattern Recognition, 44(6):1172 -1182, 2011.

[35] T. Skopal, B. Bustos. On nonmetric similarity search problems in complex domains. ACM Comput. Surv., 43(4):34:1-34:50, 2011

[36] A. Zapata, V. Menéndez, M. Prieto, C. Romero. A framework for recommendation in learning object repositories: An example of application in civil engineering. Advances in Engineering Software, 56(0):1 - 14, 2013.

[37] Cazella, S. C., Behar, P., Schneider, D., da Silva, K. K., and Freitas, R. Desenvolvendo um sistema de recomendação de objetos de aprendizagem 
baseado em competências para a educação: relato de experiências. In Anais do Simpósio Brasileiro de Informática na Educação, Rio de Janeiro, 23, 2012.

[38] R. Rocha, R. Franco, R. S. Barros, E. R. G. Dantas, R. R. Azevedo, G. A. Dias, A. S. Gomes. Amadeus recommends: um sistema de recomendação para objetos de aprendizagem. In Anais do Workshop de Informática na Escola, 1:14571460,2010

[39] M. A. S. Nunes, S. C. Cazella. O que sua personalidade revela? Fidelizando clientes web através de Sistemas de Recomendação e traços de personalidade. Minicursos do Webmedia. SBC. Capítulo, 5,2011 .

[40] M. Matuschek, T. Schlüter, S. Conrad. Measuring text similarity with dynamic time warping. In Proceedings of the 2008 international symposium on Database engineering \& applications, IDEAS '08, New York, p. 263-267, 2008. 